\title{
Effects of Various Sugars Added to Growth and Drying Media upon Thermotolerance and Survival throughout Storage of Freeze-Dried lactobacillus del brueckii ssp. bulgaricus
}

\author{
Ana S. Carvalho, ${ }^{\dagger}$ J oana Silva, ${ }^{\dagger}$ Peter Ho, ${ }^{\ddagger}$ Paula Teixeira, ${ }^{*}{ }^{\dagger}$ F . Xavier Malcata, ${ }^{\dagger}$ and \\ Paul Gibbs ${ }^{\dagger}$
}

Escola Superior de Biotecnologia, Universidade Católica Portuguesa, Rua Dr. António Bernardino de AImeida, P-4200-072 Porto, Portugal, and Departamento de Ciências de Engenharia e Tecnologia,

Escola Superior de Tecnol ogia e Gestão, I nstituto Politécnico de Viana do Castelo, Apartado 574,

P-4901-908 Viana do Castelo, Portugal

\begin{abstract}
The aim of this research effort was to investigate the role of various sugar substrates in the growth medium upon thermotolerance and upon survival during storage after freeze-drying of Lactobacillus bulgaricus. Addition of the sugars tested to the growth medium, and of these and sorbitol to the drying medium (skim milk) was investigated so as to determine whether a relationship exists between growth and drying media, in terms of protection of freeze-dried cells throughout storage. The lowest decrease in viability of L. bulgaricus cells after freeze-drying was obtained when that organism was grown in the presence of mannose. However, L. bulgaricus clearly survived better during storage when cells had been grown in the presence of fructose, lactose or mannose rather than glucose (the standard sugar in the growth medium). A similar effect could not be observed in terms of thermotolerance; in this case, the growth medium supplemented with lactose was found to yield cells bearing the highest heat resistance. Supplementation of the drying medium with glucose, fructose, lactose, mannose or sorbitol led in most cases to enhancement of protection during storage, to a degree that was growth medium-dependent.
\end{abstract}

\section{Introduction}

Lactobacillus delbruekii ssp. bulgaricus is important in a number of industrial applications and is frequently employed as a starter culture for dairy fermentations; combined with other species, it is in particular used in the industrial manufacture of yoghurt and of such cheese types as emmental, gruyère, gorgonzola, mozarella, cacciocavallo, and provolone (32). As happens with this and many other starter cultures with commercial expression, freezing and freeze-drying are commonly used for the preservation and storage of such starter microorganisms for later application by the food industry. Optimal performance of given strains must at first guarantee their potential to survive, so as to ensure eventual stabilization of their metabolic activity (4). To preserve cell viability during dehydration, several parameters should be considered; a few studies $(1,16,20,23-25)$ have provided evidence of high viability when microbial dehydration is performed in the presence of protective agents. A microorganism that has survived previous steps of freeze drying may eventually lose its viability during storage; hence, the storage stability of lactic acid bacteria (LAB) is a very important factor toward their industrial application.

Exopolysaccharide (EPS) production by L. bulgaricus has been the focus of considerable research, much of

* To whom correspondence should be addressed. Tel: +351 225580095. Fax: +351 225090351. E-mail: paula@esb.ucp.pt.

† Universidade Católica Portuguesa.

₹ Instituto Politécnico de Viana do Castelo. which has dealt with the effects of medium composition and growth conditions on the amounts of EPS produced (22). Note that EPS is a general term that refers to two types of secreted polysaccharides; one type is attached to the cell wall as a capsule (also desi gnated as capsular polysaccharide or CPS), whereas the other is produced as loose, unattached material (also termed slime EPS and hereafter referred to simply as EPS) (12). The total amount of EPS produced, as well as the capsule size, depends on the carbohydrate (which is used as carbon source) present in the growth medium $(12,18)$. It is believed that EPSs play some kind of biological role, because it is rather unlikely that bacteria use both substrate and energy for the production of useless metabolites (26). Most functions proposed for EPS are in general of a protective nature, e.g., protection against desiccation, phagocytosis and predation by protozoa, phage attack, antibiotic action, toxic compounds, and osmotic stress (31).

The present work was undertaken so as to fulfill two major goals: (I) assess the influence of various sugars added to the growth and to the drying media upon survival of $L$. bulgaricus during freeze-drying and subsequent storage, and (II) study the effect of various sugar substrates upon its thermotolerance and EPS production. An extensive literature search has indicated that this is apparently the first study on the role of various sugars, normally added only to the drying medium, when previously added also to the growth medium. 


\section{Materials and Methods}

Organism and Media. Lactobacillus del brueckii ssp. bulgaricus (hereafter simply termed L. bulgaricus) had been previously isolated from yoghurt and was obtained from the culture collection held at Escola Superior de Biotecnol ogia (Portugal). The original reference cultures were maintained in cryogenic storage at $-80^{\circ} \mathrm{C}$ on glass beads. Working cultures were grown on de Man, Rogosa, Sharp (MRS) broth (LAB M, Bury, U.K.), containing 1.5\% $(\mathrm{w} / \mathrm{w})$ agar (MRSa), as slope cultures $\left(37^{\circ} \mathrm{C}\right.$ for $24 \mathrm{~h}$ ). Slopes were stored at $4{ }^{\circ} \mathrm{C}$ and subcultured every month. MRS broth (100 mL) was inoculated in $200-\mathrm{mL}$ glass bottles from the MRSa slopes, and incubated without agitation for $24 \mathrm{~h}$ at $37{ }^{\circ} \mathrm{C}$. This culture was then inoculated at the $1 \%(\mathrm{v} / \mathrm{v})$ level, in similar glass bottles and again without agitation, into standard MRS broth $(100 \mathrm{~mL})$, i.e., MRS-glucose ( $20 \mathrm{~g} / \mathrm{L}$ glucose), and al so into modified MRS broth (100 mL) with glucose replaced by $20 \mathrm{~g} / \mathrm{L}$ fructose (MRS-fructose), by $20 \mathrm{~g} / \mathrm{L}$ lactose (MRSlactose), or by $20 \mathrm{~g} / \mathrm{L}$ mannose (MRS-mannose) and incubated at $37{ }^{\circ} \mathrm{C}$ for $24 \mathrm{~h}$ in the case of MRS-glucose, MRS-fructose, and MRS-lactose, and for $48 \mathrm{~h}$ in the case of MRS-mannose. All cells were harvested in the same growth phase (stationary), although the final concentration of cells in the fructose medium was somewhat lower than in the other media (see initial values in Figures 1 and 2).

Culture Preparation. Cells from 100-mL cultures were harvested by centrifugation at $7000 \times \mathrm{g}$ for $10 \mathrm{~min}$, washed twice by centrifugation with sterile Ringer's solution (30) (LAB M), and then resuspended in the same volume of either sterile skim milk containing $11 \%(\mathrm{w} / \mathrm{v})$ solids (LAB M) or sterile skim milk containing each of the following compounds: glucose (10 g/L), fructose (10 $\mathrm{g} / \mathrm{L})$, lactose $(10 \mathrm{~g} / \mathrm{L})$, mannose $(10 \mathrm{~g} / \mathrm{L})$, or sorbitol $(10$ $\mathrm{g} / \mathrm{L})$. Cellular suspensions were maintained for $1 \mathrm{~h}$ at room temperature $\left(20^{\circ} \mathrm{C}\right.$ ) prior to freezing at $-80^{\circ} \mathrm{C}$ for $24 \mathrm{~h}$, so as to allow for equilibration between the cells and the added compound. For each growth medium, 1-mL samples were taken and added to $9 \mathrm{~mL}$ of Ringer's solution (LAB M); suitable dilutions were then plated on MRS agar by the drop count technique (28). Three drops (20 $\mu \mathrm{L}$ each) of suitable dilutions were placed on each of three separate plates, which were examined after incubation at $37{ }^{\circ} \mathrm{C}$ for $48 \mathrm{~h}$. The experiments were repeated twice. The viable cell count prior to freeze-drying is denoted as the first data point in Figures 1 and 2.

Freeze-Drying and Storage. Samples of $20 \mathrm{~mL}$ were desiccated under vacuum (50 mTorr for $48 \mathrm{~h}$ ) in a freezedrier (Martin Christ, Osterad am Harz, Germany), at room temperature; the condenser was cooled to $-55^{\circ} \mathrm{C}$. Dried cells were stored at room temperature $\left(20^{\circ} \mathrm{C}\right)$ in closed containers under air and maintained in the dark.

E numeration of Survivors. I mmediately after freezedrying and at regular time intervals during storage, freeze-dried samples taken at random were rehydrated to the original volume with deionized water, and suitable dilutions were plated on MRS agar by the drop count technique as described before. Plates were examined after incubation at $37^{\circ} \mathrm{C}$ for $48 \mathrm{~h}$. The degree of survival was expressed in CFU/mL.

Measurement of Heat Resistance. A volume $(2 \mathrm{~mL})$ of cellular suspension in skim milk, immediately before freezing, was well mixed with $48 \mathrm{~mL}$ of Ringer's solution, previously equilibrated at $62{ }^{\circ} \mathrm{C}$. At appropriate time intervals, 1-mL samples were taken from the medium held at $62{ }^{\circ} \mathrm{C}$ and added to $9 \mathrm{~mL}$ of Ringer's solution. After serial decimal dilutions in Ringer's solution, sur-

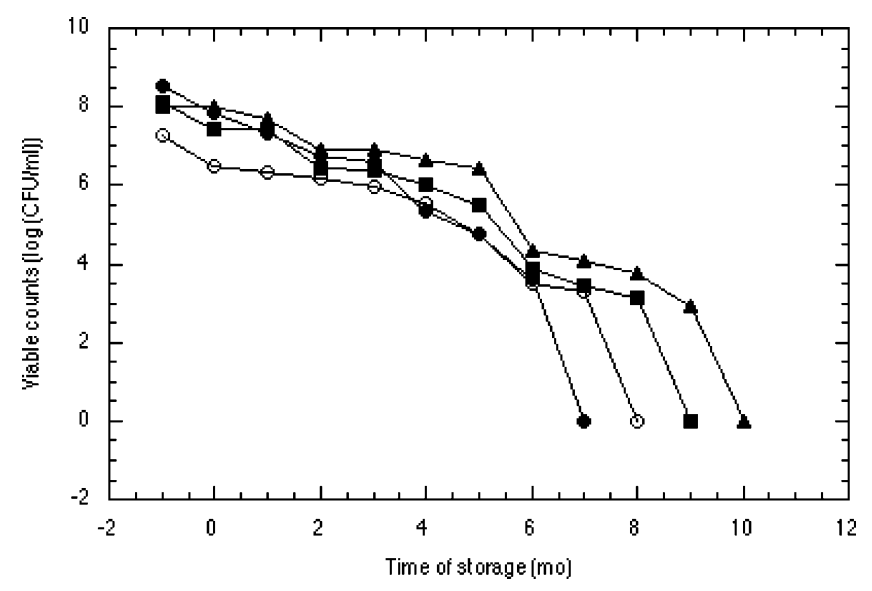

Figure 1. Effect of various growth media [( $\square)$ MRS-glucose,

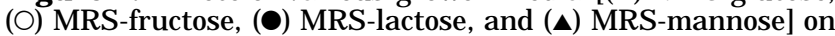
survival during freeze-drying and subsequent storage at room temperature of Lactobacillus bulgaricus. Each symbol represents the mean of three replicates from two freeze-drying experiments, in logarithmic form (six values in all); Tukey's HSD, based on $95 \%$ confidence intervals of treatment means, is equal to 0.34 for all data points. The initial point in each graph represents the viable count in each cell suspension before freezedrying.

vivors were enumerated on MRS agar by the drop count technique. Plates were examined after incubation at 37 ${ }^{\circ} \mathrm{C}$ for $48 \mathrm{~h}$. The heat resistance of an organism may be defined by its $D_{T}$ value, i.e., the time required to kill $90 \%$ of that microorganism (or spores thereof) in a sample at a given temperature $\mathrm{T}$ (in ${ }^{\circ} \mathrm{C}$ ) (13); $\mathrm{D}_{\mathrm{T}}$ values were determined by plotting the log of the number of survivors at temperature $\mathrm{T}$ versus time and calculating the slope of the best fit straight line.

Screening for Exopolysaccharide. Modified and standard MRS media were inoculated (at $1 \%$ ) with strains pregrown in MRS medium. After incubation for $24 \mathrm{~h}$ at $37^{\circ} \mathrm{C}$, ca. $20 \mathrm{~mL}$ of each cellular suspension was harvested by centrifugation in a 70-mL centrifuge tube and then heated in a boiling water bath for $10 \mathrm{~min}$ (in order to inactivate enzymes that might degrade EPS). Boiled samples were cooled to room temperature and then duly centrifuged (at $7000 \times \mathrm{g}$ for $20 \mathrm{~min}$ ). Two volumes of cold $\left(4^{\circ} \mathrm{C}\right)$ ethanol were added to one volume of culture supernatant, and the mixture was stored overnight at $4{ }^{\circ} \mathrm{C}$ so as to all ow precipitation of EPS. The precipitate was collected by centrifugation (at $7000 \times \mathrm{g}$ for $20 \mathrm{~min}$ ) and resuspended in one volume of deionized water. The total sugar concentration was determined by the phenol/sulfuric acid method (14); samples were treated with pure sulfuric acid and phenol, and then the absorbances were measured at $490 \mathrm{~nm}$. The EPS production was expressed as $\mathrm{mg} / \mathrm{L}$.

Statistical Analysis. Analysis of variance (ANOVA) of viable counts (in log CFU/mL), before and after freezedrying and at regular time intervals during storage, was carried out with the statistical program R (21). Statistical differences were considered at the 0.05 level of significance. Multiple comparison of treatment means (3 replicates $\times 2$ experiments) was done using a 95\% confidence interval based on Tukey's honestly significant difference (HSD); subsequently, mean values using the Trellis display (5) were plotted (with indication of HSD values).

\section{Results and Discussion}

A full four-factor ANOVA, encompassing replicates of freeze-drying (experimental replication), storage time, drying medium, and growth medium, was carried out. 


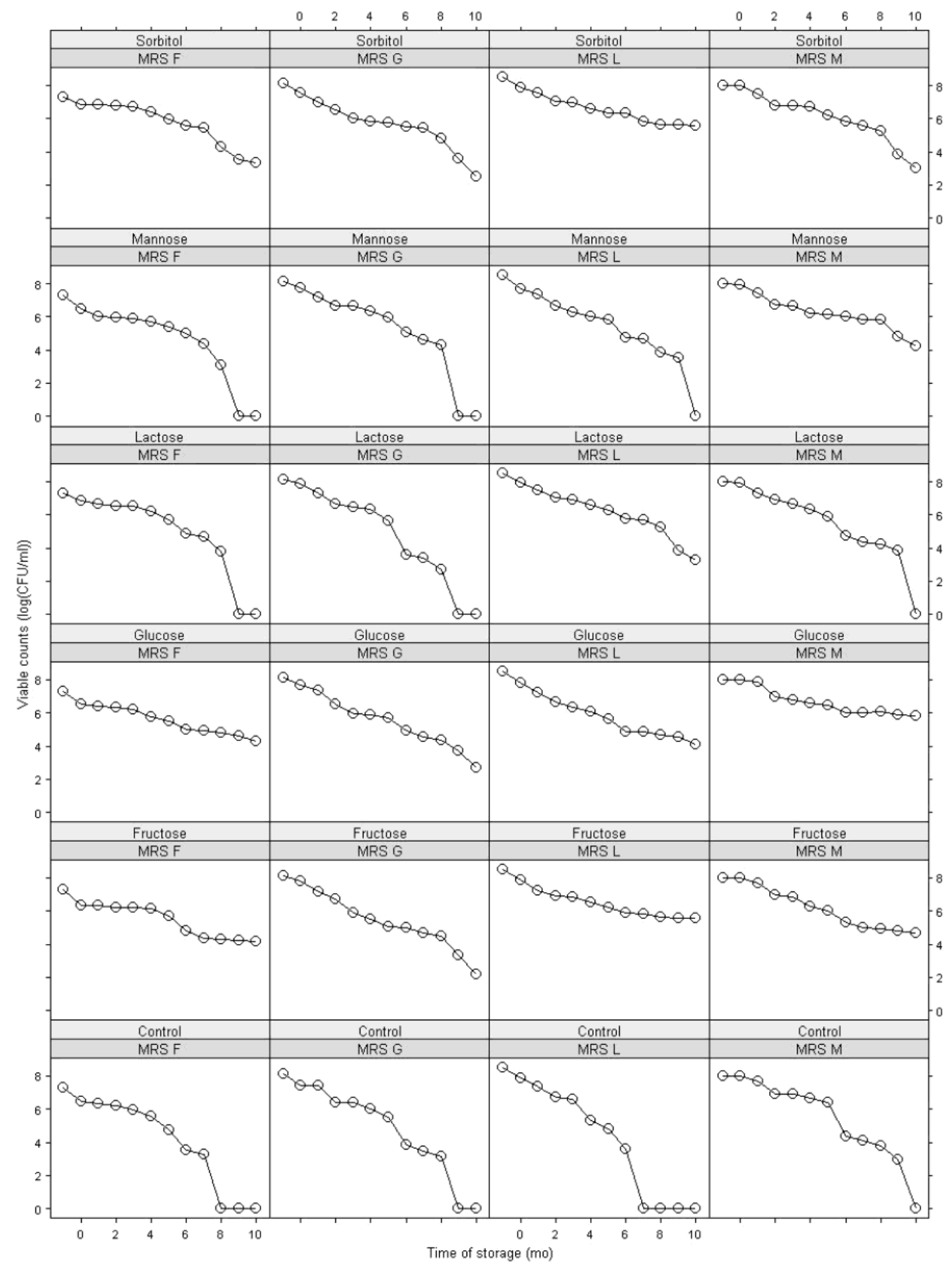

Figure 2. Effect of various drying medium compounds [skim milk (SM) (Control), SM with fructose (fructose), SM with glucose (glucose), SM with lactose (lactose), SM with mannose (mannose), and SM with sorbitol (sorbitol)] on survival during freeze-drying and subsequent storage at room temperature of Lactobacillus bulgaricus that have been grown in MRS with different sugar substrates [MRS-glucose (MRS G), MRS-fructose (MRS F), MRS-lactose (MRS L), and MRS-mannose (MRS M)]. Each symbol represents the mean of three replicates from two freeze-drying experiments, in logarithmic form (six values in all); Tukey's HSD, based on 95\% confidence intervals of treatment means, is equal to 0.34 for all data points. The initial point in each graph represents the viable count in each cell suspension before freeze-drying.

The results of that analysis are shown in Table 1. Experimental replication and its two-way interactions with the other three factors were not significant. The survival of freeze-dried L. bul garicus during storage was shown to be dependent on the drying medium ( $p<$ $0.0001)$ and on the growth medium ( $<0.0001)$. The influence of the drying medium on survival during storage depended on the specific growth medium em- 
Table 1. ANOVA Table of the Effects of Various Growth and Drying Media on Survival during Freeze-Drying and Subsequent Storage of Lactobacillus bulgaricus

\begin{tabular}{|c|c|c|c|c|}
\hline source of variation & sum of squares & degrees of freedom & mean square & F-ratio \\
\hline experimental replication (E) & 0.0272 & 1 & 0.03 & 2.93 \\
\hline storage time (ST) & 3383.6 & 11 & 307.6 & $33127.38^{a}$ \\
\hline drying medium (DM) & 275.5 & 5 & 55.1 & $5933.73^{a}$ \\
\hline growth medium (GM) & 173.2 & 3 & 57.7 & $6218.63^{a}$ \\
\hline$\widehat{E} \times \mathrm{ST}$ & 0.1 & 11 & 0.01 & 1.14 \\
\hline $\mathrm{E} \times \mathrm{DM}$ & 0.0265 & 5 & 0.005 & 0.57 \\
\hline $\mathrm{E} \times \mathrm{GM}$ & 0.0474 & 3 & 0.02 & 1.70 \\
\hline $\mathrm{ST} \times \mathrm{DM}$ & 477.7 & 55 & 8.7 & $935.38^{a}$ \\
\hline $\mathrm{ST} \times \mathrm{GM}$ & 134.0 & 33 & 4.1 & $437.34^{a}$ \\
\hline $\mathrm{DM} \times \mathrm{GM}$ & 112.7 & 15 & 7.5 & $809.34^{a}$ \\
\hline $\mathrm{E} \times \mathrm{ST} \times \mathrm{DM}$ & 0.2 & 55 & 0.004 & 0.43 \\
\hline $\mathrm{E} \times \mathrm{ST} \times \mathrm{GM}$ & 0.1 & 33 & 0.003 & 0.35 \\
\hline $\mathrm{E} \times \mathrm{DM} \times \mathrm{GM}$ & 0.1 & 15 & 0.006 & 0.72 \\
\hline $\mathrm{ST} \times \mathrm{DM} \times \mathrm{GM}$ & 212.1 & 165 & 1.3 & $138.45^{\mathrm{a}}$ \\
\hline $\mathrm{E} \times \mathrm{ST} \times \mathrm{DM} \times \mathrm{GM}$ & 0.4 & 165 & 0.003 & 0.28 \\
\hline residuals & 5.3 & 576 & 0.009 & \\
\hline
\end{tabular}

a $p<0.0001$.

ployed, as concluded from the significant $(p<0.0001)$ two-way interaction associated therewith. To compare the differences between mean values of survival at any specific point during storage, multiple comparison of means was done on the basis of Tukey's HSD. (Because of the statistical significance of the 2-way interactions between added compound and storage time, multiple comparisons should be conducted using their individual treatment means and not their factor level means (29).)

Although it might be argued that the acidifying activity, rather than the number of cells that have undergone the preservation process, should have been elected as dependent variable, note that it is common practice in the literature to proceed in the latter way. In any case, the method devel oped by Martos et al. (27), coupled with the contribution by Carvalho et al. (9), has proven that the number of cells obtained by the plate count is essentially equivalent to the number of active cells detected by impedimetric methods (as in our case). On the other hand, the originality of this research effort derives from the assessment of the effects of addition of various sugars to the growth medium itself, which are regularly added only to the drying medium. Furthermore, the sugar source in the growth medium also plays a critical role in survival during freeze-drying and subsequent storage, which interacts with each additive to the drying medium.

The original data in logarithmic form, rather than the reduction in viable numbers, was represented in order to maximize the informational content of Figures 1 and 2. In the case of the medium MRS-fructose, the number of cells in the final culture was lower than in the case of MRS containing other sugars (probably as a result of increased production of EPS); however, the physiological state at which bacteria were harvested was similar to that prevailing in the other media.

Effect of Growth Medium. Freeze-Drying and Storage. The lowest decrease in viability of $L$. bul garicus cells after freeze-drying was obtained when that organism was grown in MRS-mannose (Table 2); the same tendency was observed during storage. The log reduction observed in MRS-mannose indicated that this sugar would be effective toward growth of L. bulgaricus cells if the objective were the preservation of viability during storage; there were no significant differences between cell survival during dried storage when growth was in either standard MRS-glucose, MRS-fructose, or MRS-lactose. These observations indicate that a sugar other than glucose in the standard MRS should be used in order to
Table 2. Effect of Various Sugar Substrates Present in Growth Media on Survival (in $\log (\mathrm{CFU} / \mathrm{mL}$ )) of Lactobacillus bulgaricus during Freeze-Dryinga

\begin{tabular}{|c|c|c|c|c|c|c|}
\hline & \multicolumn{3}{|c|}{ before freeze-drying } & \multicolumn{3}{|c|}{ after freeze-drying } \\
\hline & lower & mean & upper & lower & mean & upper \\
\hline MR & 7.79 & 8. & 8.48 & 7.11 & 7.45 & 7.79 \\
\hline MRS-fructose & 6.93 & 7.27 & 7.61 & 6.13 & 6.48 & 6.82 \\
\hline MR & 8.16 & 8.51 & 8.85 & 7.53 & 7.87 & 8.21 \\
\hline MRS-mannose & 7.67 & 8.02 & 8.36 & 7.61 & 7.95 & 8.29 \\
\hline
\end{tabular}

a Each value represents the mean of three replicates from two freeze-drying experiments (six values in all); Tukey's HSD, based on $95 \%$ confidence intervals of treatment means, is equal to 0.34 for all data points.

extend the viability of the dried cells during storage at room temperature. The important interactions found between the growth medium and the drying medium will be discussed later.

Our observations strongly suggest that growth in the presence of various sugar substrates produces cells with different morphological and physiological traits, which are consubstantiated in distinct resistances to the various stress treatments tested. Studies by Hofvendahl et al. (19) showed that other compounds are formed by homofermenters in addition to lactic acid, during growth on sugars other than gl ucose. Some homofermentative LAB were found (33) to produce small amounts of mannitol, which in most cases remained inside the cell. It is, therefore, possible that mannitol (or even other compounds) is produced during fermentation on some of the sugars tested, and that this compound might be responsible for the distinct survival behaviors during storage of freeze-dried L. bulgaricus. The protective effect of mannitol as a compatible solute, in microorganisms subjected to low water activity, may result in enhanced viability of the starter cultures in dried form.

Thermotolerance I nspection of Table 3 indicates that cell thermotolerance depends on the growth medium utilized; the highest thermotolerance was obtained when cells were grown in MRS-lactose. The $D_{62}$ value for stationary-phase cells of L. bulgaricus in MRS-lactose $(19.0 \pm 1.75 \mathrm{~min})$ was approximately 2 -fold those found for cells grown in other media, MRS-glucose $(8.55 \pm 0.17$ $\mathrm{min})$, MRS-fructose $(8.27 \pm 0.42 \mathrm{~min})$, and MRS-mannose $(9.26 \pm 0.81 \mathrm{~min})$ (see Table 3$)$. Variation in the specific rates of exponential growth, sugar consumption, and product formation, which depend on the sugar composition of the medium, could lead to cells with distinct characteristics, and consequently different resistances to 
Table 3. Effect of Various Sugar Substrates Present in Growth Media on Thermotolerance of Lactobacillus bulgaricusa

\begin{tabular}{lrcr}
\hline & \multicolumn{3}{c}{$\mathrm{D}_{62}$ value (min) } \\
\cline { 2 - 4 } growth medium & Iower & mean & upper \\
\hline MRS-glucose & 8.38 & 8.55 & 8.72 \\
MRS-fructose & 7.85 & 8.27 & 8.69 \\
MRS-lactose & 17.25 & 19.0 & 20.75 \\
MRS-mannose & 8.45 & 9.26 & 10.07
\end{tabular}

a Each value represents the mean of three replicates from two individual experiments (six values in all).

heat stress. In addition, the distinct growth kinetics (data not shown) in each of the media tested might impose different degrees of stress, thereby inducing synthesis of different shock proteins. Studies by Even et al. (15) unfolded differences in physiological behavior when Lactococcus lactis was grown in the presence of two different sugars, and Annous et al. (3) claimed that nutrient sources other than glucose, when present in the growth medium, affect the membrane fluidity via alteration of its fatty acid content-and it is known that membrane fatty acid composition plays a critical role upon thermal resistance.

Our experimental results also demonstrated that sugar substrates that enhance resistance to storage in the dried state do not coincide with those that promote cell survival during heating. Similar results were obtained by Carpenter et al. (7), who suggested that the means by which carbohydrates stabilize dried proteins is fundamentally different from that observed in the aqueous and in the frozen state.

EPS Synthesis. EPS production is an important industrial trait of $\mathrm{L}$. bulgaricus, namely, for yoghurt quality, although the amounts of EPS produced thereby are often small (10). As observed in Table 4, EPS production by the L. bulgaricus strain tested was affected by composition of the growth medium. Higher EPS production was recorded when the cells were grown in the medium containing fructose; the amount of EPS produced in MRS-mannose was significantly lower than that in MRS-fructose, but higher than that in MRSglucose or MRS-lactose.

It was suggested (31) that EPS could protect the cells against dehydration, since it may exhibit properties similar to those described for glass-forming polymers (6). Our results did not, however, unfold any significant correlation between resistance during storage of freezedried cells and amount of EPS produced.

Effect of Drying Medium. Freeze-Drying and Storage. The additives tested in this study for their role as protective agents may be divided into two groups: (i) glucose, fructose, lactose, and mannose, which are metabolized by L. bulgaricus and which were used as sugar sources in the growth medium; and (ii) sorbitol, which was found to be the most effective protectant for both $L$. plantarum and $L$. rhamnosus during storage after freezedrying (8).

Inspection of Figure 2 indicates that supplementing skim milk with protective agents often enhanced its intrinsic protective effect during storage, to different degrees depending on the compound added. For all growth media, glucose, fructose and sorbitol were found to be good protectants, but again the degree of protection was dependent on the nature of the growth medium. Note that the storage conditions elected for our study were deliberately poor (i.e., in air at room temperature), so a 2-3 log cycle reduction during 10 months obtained for
Table 4. Effect of Various Sugar Substrates Present in Growth Media on Exopolysaccharide Production by Lactobacillus bulgaricus ${ }^{a}$

\begin{tabular}{lrcc}
\hline & \multicolumn{3}{c}{ EPS (mg/L) } \\
\cline { 2 - 4 } growth medium & Iower & mean & upper \\
\hline MRS-glucose & 95.0 & 102.6 & 110.2 \\
MRS-fructose & 147.8 & 152.6 & 157.4 \\
MRS-lactose & 104.0 & 106.6 & 109.2 \\
MRS-mannose & 114.0 & 120.7 & 127.4
\end{tabular}

a Each value represents the mean of three replicates from two individual experiments (six values in all).

some of the combinations tested was considered sufficient for our objectives. For lactose and mannose, protection was even more dependent on the growth medium, especially by the end of the storage period tested; those compounds had a positive effect on survival during storage only if growth occurred in the presence of the same sugar. Similar results were reported by Abadias et al. (2), who demonstrated a significant increase in viability of Candida sake cells when the same solution tested as protectant was used to rehydrate dried samples.

When L. bulgaricus cells were grown in MRS-glucose, there were no significant differences in survival during storage when skim milk was supplemented with glucose, fructose, or sorbitol (see Figure 2), but cell survival was enhanced (compared to the control). On the other hand lactose and mannose were shown to be ineffective in protecting cells during storage. A similar behavior was found for cells that had been grown in MRS-fructose, but the degree of protection was much greater. For cells grown in MRS-lactose and MRS-mannose, addition of glucose, fructose or sorbitol to skim milk resulted in extended survival. However, in the case of MRS-lactose, L. bulgaricus exhibited significantly higher survival rates during storage in the dried form when lactose was added to skim milk than when grown in MRS supplemented with other sugars. Mannose in skim milk also seemed to improve survival when cells had been grown in the presence of this sugar, but not when growth occurred in the presence of the other sugars considered (see Figure 2).

The differences in cell survival observed in this study indicate that certain additives are more effective than others in protecting L. bulgaricus throughout storage; maximum protection of cells of $L$. bulgaricus during storage was indeed achieved with glucose, fructose, and sorbitol, even though the effect of these compounds was not similar in the various growth media tested. It has been reported (11) that sugars can replace structural water molecules in membranes after dehydration, and thus prevent unfolding and aggregation of proteins by hydrogen bonding with polar groups of proteins. Increased residual activity and viability during drying, and subsequent storage following addition of sorbitol to the drying medium, had been previously reported $(1,8,24)$ for various organisms, including L. bulgaricus. Mechanisms proposed in attempts to rationalize protection by sorbitol during storage include prevention of membrane damage by interaction with membrane components and/ or prevention of lipid oxidation owing to its antioxidant properties $(25,33)$, as well as stabilization of protein functionality and structure (34).

No significant differences were observed in our study pertaining to viability of cells during drying in the presence or absence of glucose (or fructose, for that matter); however, survival during storage was higher in their presence for all growth media tested. Abadias et 
al. (1) also claimed that glucose was not effective in preserving $C$. sakecells during lyophilization. Linders et al. (24) reported that addition of fructose to the drying medium did not increase the residual activity after fluidized bed-drying of L. plantarum; however, Carvalho et al. (8) found that fructose had a positive effect on the survival of freeze-dried L. plantarum and L. rhamnosus during storage.

The characteristics of the transporters involved in the uptake of sugars, which may result in efficient or inefficient import of the sugar, could thus lead to differences in their efficiency as protectants during storage in the dried state; it has been reported (10) that for L. bulgaricus strain CNRZ397, glucose, mannose, and fructose are imported via the PTS-specific enzyme ElIMan, whereas lactose is transported via lactose permease (LaCS). It was observed that the level of enzymes involved in such a transport was affected by the carbon source present in the growth medium (17). Both factors could provide possible explanations for the results presented and elucidate the growth medium dependence observed in the case of lactose and mannose.

\section{Conclusions}

The results of this study demonstrate that thermotolerance of L. bulgaricus, survival after freeze-drying, and EPS production depend on the sugars added to the growth medium and, in the case of freeze-drying, also on the sugars added to the drying medium. Although cause and effect mechanisms require further investigation, our results already indicate the importance of the growth and drying medium on survival during storage of freeze-dried L. bulgaricus. Moreover, our research effort has shown that treatments that enhance resistance to freeze-drying (and subsequent storage) and acquisition of thermotolerance are not necessarily coincident.

\section{Acknowledgment}

This work received financial support through project PRAXIS/P/BIO/12147/1998 (FCT, Portugal), coordinated by P.T. and P.G. Financial support for authors A.S.C. and J.S. was provided by PhD fellowships, PRAXIS XXI/BD/ 18152/98 and PRAXIS XXI/BD/197131/99, respectively (FCT).

\section{References and Notes}

(1) Abadias, M.; Benabarre, A.; Teixidó, N.; Usall, J .; Viñas, I. E ffect of freeze-drying and protectants on viability of biocontrol yeast Candida sake. Int. J . Food Microbiol. 2001, 65, 173-182.

(2) Abadias, M.; Teixidó, N.; Usall, J .; Benabarre, A.; Viñas, I. Viability, efficacy, and storage of freeze-dried biocontrol agent Candida sake using different protective and rehydration media. J . Food Prot. 2001, 64, 856-861.

(3) Annous, B. A.; Kozempel, M. F.; Kurantz, M. J . Changes in membrane fatty acid composition of Pediococcus sp. strain NRRL B-2354 in response to growth conditions and its effect on thermal resistance. Appl. Environ. Microbiol. 1999, 65, 2857-2862.

(4) Bâati, L.; Fabre-Ga, C.; Auriol, D.; Blanc, P. J . Study of the cryotolerance of Lactobacillus acidophilus: effect of culture and freezing conditions on the viability and cellular protein levels. Int. J . Food Microbiol. 2000, 59, 241-247.

(5) Becker, R. A.; Cleveland, W. S.; Shyu, M. J. The visual design and control Trellis display. J . Comput. Graphics Stat. 1996, 5, 123-155.
(6) Billi, D.; Potts, M. Life and death of dried prokaryotes. Res. Microbiol. 2002, 153, 7-12.

(7) Carpenter, J . F.; Arakawa, T.; Crowe, J . H. Interactions of stabilizing additives with proteins during freeze-thawing and freeze-drying. Dev. Biol. Stand. 1991, 74, 225-239.

(8) Carvalho, A. S.; Silva, J .; Ho, P.; Teixeira, P.; Malcata, F. X.; Gibbs, P. Effect of additives on survival of freeze-dried Lactobacillus plantarum and Lactobacillus rhamnosus during storage. Biotechnol. Lett. 2002, 24, 1587-1591.

(9) Carvalho, A. S.; Silva, J .; Ho, P.; Teixeira, P.; Malcata, F. X.; Gibbs, P. I mpedimetric method for estimating the residual activity of freeze-dried Lactobacillus del bruekii ssp. bulgaricus. Int. Dairy J . 2003, 13, 463-468.

(10) Chervaux, C.; Ehrlich, S. D.; Maguin, E. Physiological study of Lactobacillus delbruekii subsp. bulgaricus strains in a novel chemically defined medium. Appl. Environ. Microbiol. 2000, 66, 5306-5311.

(11) Costa, E.; Usall, J .; Teixidó, N .; Garcia, N.; Viñas, I. Effect of protective agents, rehydration media and initial cell concentration on viability of Pantoea agglomerans strain CPA-2 subjected to freeze-drying. J . Appl. Microbiol. 2000, 89, 193-800.

(12) Degeest, B.; Vaningelgem, F.; de Vuyst, L. Microbial physiology, fermentation kinetics, and process engineering of heteropolysaccharide production by lactic acid bacteria. Int. Dairy J . 2001, 11, 747-757.

(13) Desmond, C.; Stanton, C.; Fitzgerald, G. F.; Collins, K.; Ross, R. P. Environmental adaptations of probiotic lactobacilli towards improvement of performance during spray drying. Int. Dairy J . 2001, 11, 801-808.

(14) Dubois, M.; Gilles, K. A.; Hamilton, J . K.; Rebers, P. A.; Smith, F. Colorimetric method for determination of sugars and related substances. Anal. Chem. 1956, 28, 350-356.

(15) Even, S.; Lindley, N. D.; Cocaign-Bousquet, M. Molecular physiology of sugar catabolism in Lactococcus lactis I L 1403. J. Bacteriol. 2001, 183, 3817-3824.

(16) Font de Valdéz, G.; de Giori, G.; de Ruiz Holgado, A. P.; Oliver, G. Protective effect of adonitol on lactic acid bacteria subjected to freeze-drying. Appl. Environ. Microbiol. 1983, 45, 302-304.

(17) Garro, M. S.; Font de Valdez, G.; Olivier, G.; de Giori, G. $\mathrm{S}$. I nfluence of carbohydrates on the $\alpha$-galactosidase activity of Lactobacillus fermentum. Curr. Microbiol. 1996, 33, 302305.

(18) Hassan, A. N.; Frank, J . F.; Shalabi, S. I. Factors affecting capsule size and production by lactic acid bacteria used as dairy starter cultures. I nt. J . Food Microbiol. 2001, 64, 199203.

(19) Hofvendahl, K.; Hahn-Hägerdal, B. Factors affecting the fermentative lactic acid production from renewable resources. Enzyme Microb. Technol. 2000, 26, 87-107.

(20) Hubalék, Z. Cryopreservation of Microorganisms; Academia: Prague, Czech Republic, 1996.

(21) Ihaka, R.; Gentleman, R. R. A language for data analysis and graphics. J. Comput. Graphics Stat. 1996, 5, 299-314.

(22) Kimmel, S. A.; Roberts, R. F. Development of a growth medium suitable for exopolysaccharide production by Lactobacillus de brueckii ssp. bulgaricus RR. Int. J . Food Mi crobiol. 1998, 40, 87-92.

(23) Leslie, S. B.; Israeli, E.; Lighthart, B.; Crowe, J . H.; Crowe, L. M. Trehalose and sucrose protect both membranes and proteins in intact bacteria during drying. Appl. Environ. Microbiol. 1995, 61, 3592-3597.

(24) Linders, L. J . M.; Wolkers, W. F.; Hoekstra, F. A.; van't Riet, K. Effect of added carbohydrates on membrane phase behaviour and survival of dried Lactobacillus plantarum. Cryobiology 1997, 35, 31-40.

(25) Linders, L. J . M.; de J ong, G. I. W.; Meerdink, G.; van't Riet, K. Carbohydrates and the dehydration inactivation of Lactobacillus plantarum: the role of moisture distribution and water activity. J . Food Eng. 1997, 31, 237-250.

(26) Looijesteijn, P. J .; Trapet, L.; de Vries, E.; Abee, T.; Hugenholtz, J. Physiological function of exopolysaccharides produced by Lactococcus lactis. Int. J . Food Microbiol. 2001, $64,71-80$. 
(27) Martos, G. I.; Ruiz-Holgado, A. P.; Olivier, G.; de Valdez, G. F. Use of conductimetry to evaluate Lactobacillus debruekii ssp. bulgaricus subjected to freeze-drying. Milchwissenshaft 1999, 54, 128-130.

(28) Miles, A. A.; Misra, S. S. The estimation of the bactericidal power of blood. J . Hyg. 1938, 38, 732-749.

(29) Neter, J .; Kutner, M. H.; Nachtsheim, C.; Wasserman, W. Applied Linear Models; Irwin: Chicago, 1996.

(30) Ringer, S. Concerning the influence exerted by each of the constituents of the blood on the contraction of the ventricle. J . Physiol. 1880-1882, 3, 380-393.

(31) Ruas-Madiedo, P.; Hugenholtz, J .; Zoon, P. An overview of the functionality of exopolysaccharides produced by lactic acid bacteria. Int. Dairy J . 2002, 12, 163-171.
(32) Teixeira, P. Lactobacillus: Lactobacillus bulgaricus. In Encyclopedia of F ood Microbiology; Robinson, R. K., Batt, C. A., Patel, P. D., Eds.; Academic Press: London, 1999; pp 1144-1151.

(33) Wisselink, H. W.; Weusthuis, R. A.; Eggink, G.; Hugenholtz, J .; Grobben, G. J . Mannitol production by lactic acid bacteria: a review. Int. Dairy J . 2002, 12, 151-161.

(34) Yoo, B.; Lee, C. M. Thermoprotective effect of sorbitol on proteins during dehydration. J . Agric. Food Chem. 1993, 41, 190-192.

Accepted for publication September 25, 2003.

BP034165Y 